\title{
Neural-Network-Based Motor Rolling Bearing Fault Diagnosis
}

\author{
Bo Li, Student Member, IEEE, Mo-Yuen Chow, Senior Member, IEEE, Yodyium Tipsuwan, Student Member, IEEE, \\ and James C. Hung, Fellow, IEEE
}

\begin{abstract}
Motor systems are very important in modern society. They convert almost $60 \%$ of the electricity produced in the U.S. into other forms of energy to provide power to other equipment. In the performance of all motor systems, bearings play an important role. Many problems arising in motor operations are linked to bearing faults. In many cases, the accuracy of the instruments and devices used to monitor and control the motor system is highly dependent on the dynamic performance of the motor bearings. Thus, fault diagnosis of a motor system is inseparably related to the diagnosis of the bearing assembly. In this paper, bearing vibration frequency features are discussed for motor bearing fault diagnosis. This paper then presents an approach for motor rolling bearing fault diagnosis using neural networks and time/frequency-domain bearing vibration analysis. Vibration simulation is used to assist in the design of various motor rolling bearing fault diagnosis strategies. Both simulation and real-world testing results obtained indicate that neural networks can be effective agents in the diagnosis of various motor bearing faults through the measurement and interpretation of motor bearing vibration signatures.
\end{abstract}

Index Terms-Bearing vibration, fault diagnosis, frequency domain, neural network, time domain.

\section{INTRODUCTION}

D UE TO THE close relationship between motor system development and bearing assembly performance, it is difficult to imagine the progress of modern rotating machinery without consideration of the wide application of bearings. In addition, the faults arising in motors are often linked with bearing faults. In many instances, the accuracy of the instruments and devices used to monitor and control the motor system is highly dependent on the dynamic performance of bearings.

Bearing vibration can generate noise and degrade the quality of a product line which is driven by a motor system. Heavy bearing vibration can even cause the entire motor system to function incorrectly, resulting in downtime for the system and economic loss to the customer. Proper monitoring of bearing vibration levels in a motor system is highly cost effective in minimizing maintenance downtime - both by providing advance warning and lead time to prepare appropriate corrective actions, and by ensuring that the system does not deteriorate

Manuscript received January 15, 1999; revised March 24, 2000. Abstract published on the Internet July 1, 2000. This work was supported by the National Science Foundation under Grant ECS-9521609 and Grant ECS-9610509.

B. Li, M.-Y. Chow, and Y. Tipsuwan are with the Department of Electrical and Computer Engineering, North Carolina State University, Raleigh, NC 27695-7911 USA (e-mail: bli@eos.ncsu.edu; chow@eos.ncsu.edu; ytipsuw@unity.ncsu.edu).

J. C. Hung is with the Department of Electrical and Computer Engineering, University of Tennessee, Knoxville, TN 37996-2100 USA (e-mail: jhung@utk.edu).

Publisher Item Identifier S 0278-0046(00)08828-6. to a condition where emergency action is required. Thus, it is important to include bearing vibration diagnosis into the scheme of motor system fault diagnosis [1]-[3].

Signals from vibration sensors are usually measured and compared with reference measurements in order to interpret bearing conditions. The methods used to analyze these signals include probabilistic analysis [4], [5], frequency analysis [4]-[13], time-domain analysis [4], [5], and finite-element analysis [14]. Among these methods, the frequency analysis approach is the most popular one. This popularity is most probably due to the availability of Fourier transform technique, as characteristics of vibration signals are more easily noticed in the frequency domain rather than in the time domain. The frequency analysis technique involves frequency analysis of the vibration signal and further processing of the resulting spectrum to obtain clearly defined diagnosis information [15], [16]. Among the methods that use frequency analysis are the bearing defect frequencies analysis method [15], [16], high-frequency shock pulse and friction forces method [5], [15], [16], and enveloped spectrum method [15], [16]. In the category of time-domain analysis technique there are the time-series averaging method [16], the signal enveloping method [16], the Kurtosis method [16], and the spike energy method [16].

In the motor bearing fault diagnosis process, as shown in Fig. 1, the sensors collect time domain vibration signals. The fast Fourier transform (FFT) technique is then employed to convert the time-domain signals into frequency-domain signals, which can provide salient features for the diagnosis of the bearing condition. The designed fault diagnosis system can use both time-domain and frequency-domain signals to perform motor bearing fault diagnosis.

In this paper, neural networks are applied to motor bearing fault diagnosis. The bearing vibration frequency features and time-domain characteristics are applied to a neural network to build an automatic motor bearing fault diagnosis machine. Neural networks have a proven ability in the area of nonlinear pattern classification. After being trained, they contain expert knowledge and can correctly identify the different causes of bearing vibration. The capacity of artificial neural networks to mimic and automate human expertise is what makes them ideally suited for handling nonlinear systems. Neural networks are able to learn expert knowledge by being trained using a representative set of data [2], [3], [17]-[19]. At the beginning of a neural network's training session, the neural network fault detector's diagnosis of the motor's condition will not be accurate. An error quantity is measured and used to adjust the neural network's internal parameters in order to produce a more accurate output decision. This process is repeated until a suitable error is achieved. Once the network is sufficiently trained and the 


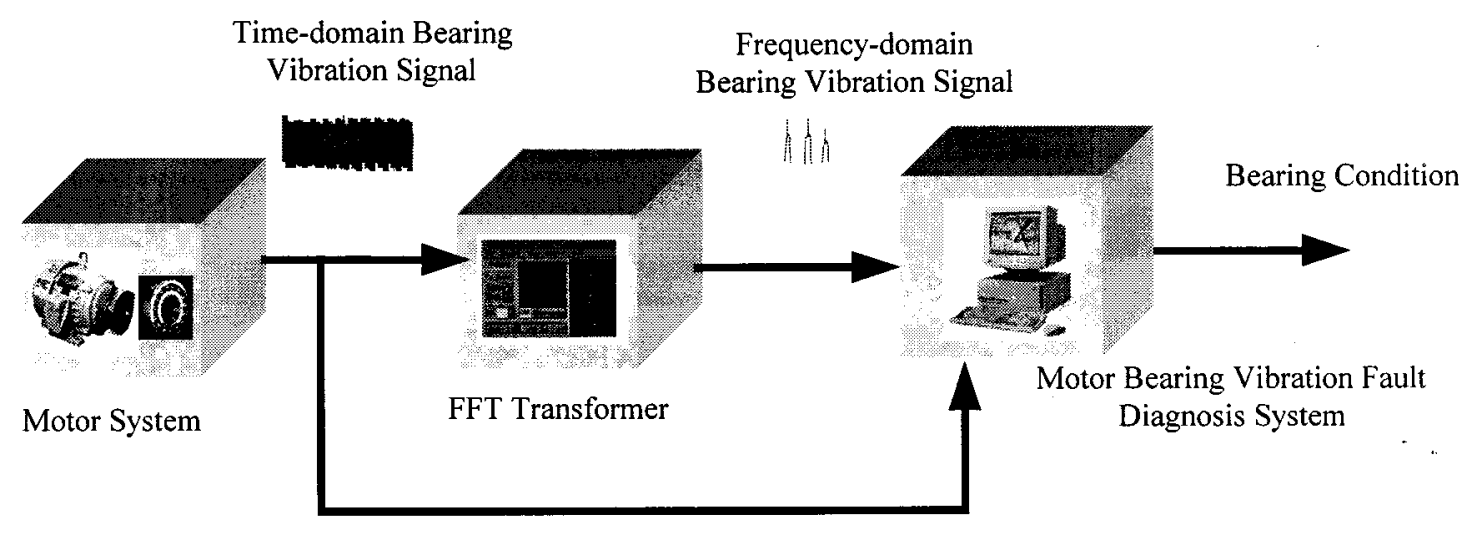

Fig. 1. General flow of signals in a typical motor bearing fault detection process.

parameters have been saved, the neural network contains all the necessary knowledge to perform the fault detection. This paper presents the design of the neural network diagnosis algorithm. Both simulation and actual experimental results indicate that a neural network bearing vibration diagnosis algorithm can give accurate information about the condition of the motor bearing based on appropriately monitored vibration data.

\section{Motor Bearing Vibration Frequency Features}

Since most bearing vibrations are periodical movements, it is easy to extract vibration features from the frequency domain using the powerful and popular FFT technique. Many publications have studied the frequency features of rolling bearing vibration [4]-[7], [20]-[22]. Generally, rolling bearings consist of two concentric rings, called the inner raceway and outer raceway, with a set of rolling elements running in their tracks. Standard shapes of rolling elements include the ball, cylindrical roller, tapered roller, needle roller, and symmetrical and unsymmetrical barrel roller [7]. Typically, the rolling elements in a bearing are guided in a cage that ensures uniform spacing and prevents mutual contact.

There are five basic motions that are used to describe the dynamics of bearing elements, with each movement having a corresponding frequency [4]-[8], [12], [21]. These five frequencies are denoted as the shaft rotational frequency $\left(F_{S}\right)$, the fundamental cage frequency $\left(F_{C}\right)$, the ball pass inner raceway frequency $\left(F_{B P I}\right)$, the ball pass outer raceway frequency $\left(F_{B P O}\right)$, and the ball rotational frequency $\left(F_{B}\right)$. These frequencies are illustrated in Fig. 2.

Fig. 3 describes several important variables that will be used in later sections. $V_{i}, V_{c}$, and $V_{o}$ represent the linear velocities of the inner raceway, ball center, and outer raceway, respectively. $D_{b}$ is the ball diameter, $D_{c}$ is the bearing cage diameter measured from one ball center to the opposite ball center, and $\theta$ is the contact angle of the bearing.

\section{A. Shaft Rotational Frequency $\left(F_{S}\right)$}

As bearings are often used to form a bearing-rotor system, the speed of the rotor (or shaft) $F_{S}$ is very important to the movements of bearings. All other frequencies are a function of this frequency.

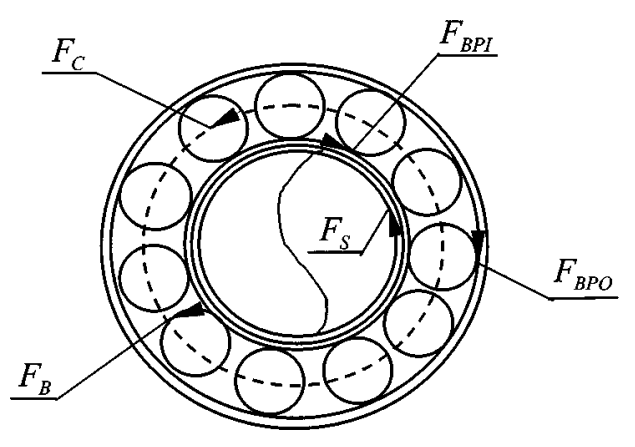

Fig. 2. Basic frequencies in a bearing.

\section{B. Fundamental Cage Frequency $\left(F_{C}\right)$}

The fundamental cage frequency $F_{C}$ is related to the motion of the cage. It can be derived from the linear velocity of a point on the cage $V_{c}$, which is the mean of the linear velocities of the inner raceway $V_{i}$, and the outer raceway $V_{o}$, i.e., $V_{c}=\left(V_{i}+V_{o}\right) / 2$. When $V_{c}$ is divided by the radius of the cage $r_{c}=\left(D_{c} / 2\right)$, we can get the fundamental cage frequency $F_{C}$. Thus, $F_{C}$ can be written as [5]-[7], [13], [23]:

$$
F_{C}=\frac{V_{c}}{r_{c}}=\frac{V_{i}+V_{o}}{D_{c}}
$$

Sometimes, it is more convenient to represent the linear velocities $V_{i}$ and $V_{o}$ as their respective rotational frequencies $F_{i}$ and $F_{o}$ multiplied by their corresponding radii $r_{i}=r_{c}-\left(D_{b} \cos \theta / 2\right)$ and $r_{o}=r_{c}+\left(D_{b} \cos \theta / 2\right)$. Thus, $F_{C}$ can be further expressed as

$$
\begin{aligned}
F_{C} & =\frac{V_{i}+V_{o}}{D_{c}}=\frac{F_{i} r_{i}+F_{o} r_{o}}{D_{c}} \\
& =\frac{1}{D_{c}}\left(F_{i} \frac{D_{c}-D_{b} \cos \theta}{2}+F_{o} \frac{D_{c}+D_{b} \cos \theta}{2}\right) .
\end{aligned}
$$

\section{Ball Pass Inner Raceway Frequency $\left(F_{B P I}\right)$}

The ball pass inner raceway frequency $F_{B P I}$ indicates the rate at which the balls pass a point on the track of the inner raceway. The value of $F_{B P I}$ is equal to the number of bearing balls $N_{B}$ multiplied by the difference between the fundamental 

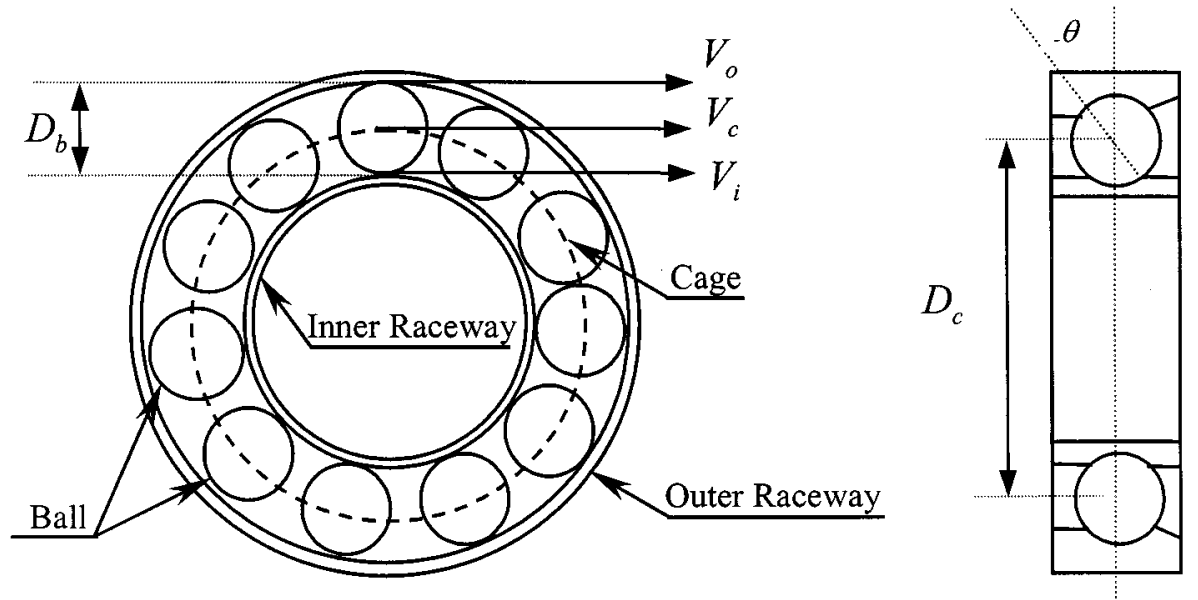

Fig. 3. Structure of a ball bearing and the definition of each variable.

cage frequency $F_{C}$ and the inner raceway frequency $F_{i}[5]-[7]$, [13], [23]

$$
\begin{aligned}
F_{B P I} & =N_{B}\left|F_{C}-F_{i}\right| \\
& =N_{B}\left|\frac{F_{i} r_{i}+F_{o} r_{o}}{D_{c}}-F_{i}\right| \\
& =N_{B}\left|\frac{F_{i}\left(r_{c}-\frac{D_{b} \cos \theta}{2}\right)+F_{o}\left(r_{c}+\frac{D_{b} \cos \theta}{2}\right)}{D_{c}}-F_{i}\right| \\
& =\frac{N_{B}}{2}\left|\left(F_{i}-F_{o}\right)\left(1+\frac{D_{b} \cos \theta}{D_{c}}\right)\right| .
\end{aligned}
$$

\section{Ball Pass Outer Raceway Frequency $\left(F_{B P O}\right)$}

Similar to the ball pass inner raceway frequency $F_{B P I}$, the ball pass outer raceway frequency $F_{B P O}$ is defined as the rate at which the balls pass a point on the track of the outer raceway. The value of $F_{B P O}$ is a function of the number of bearing balls $N_{B}$ and the difference between the outer raceway frequency $F_{o}$ and the fundamental cage frequency $F_{C}[5]-[7]$, [13], [23]

$$
\begin{aligned}
F_{B P O} & =N_{B}\left|F_{C}-F_{o}\right| \\
& =N_{B}\left|\frac{F_{i} r_{i}+F_{o} r_{o}}{D_{c}}-F_{o}\right| \\
& =N_{B}\left|\frac{F_{i}\left(r_{c}-\frac{D_{b} \cos \theta}{2}\right)+F_{o}\left(r_{c}+\frac{D_{b} \cos \theta}{2}\right)}{D_{c}}-F_{o}\right| \\
& =\frac{N_{B}}{2}\left|\left(F_{i}-F_{o}\right)\left(1-\frac{D_{b} \cos \theta}{D_{c}}\right)\right| .
\end{aligned}
$$

\section{E. Ball Rotational Frequency $\left(F_{B}\right)$}

The ball rotational frequency $F_{B}$ is the rate of rotation of a ball about its own axis in a bearing. This frequency can be calculated from either the ball pass inner raceway frequency $F_{B P I}$ or ball pass outer raceway frequency $F_{B P O}$. Both situations will give the same result [5]-[7], [13], [23]

$$
\begin{aligned}
F_{B} & =\left|\left(F_{i}-F_{C}\right) \frac{r_{i}}{r_{b}}\right|=\left|\left(F_{o}-F_{C}\right) \frac{r_{o}}{r_{b}}\right| \\
& =\frac{D_{c}}{2 D_{b}}\left|\left(F_{i}-F_{o}\right)\left(1-\frac{D_{b}^{2} \cos ^{2} \theta}{D_{c}^{2}}\right)\right|
\end{aligned}
$$

where $r_{b}$ is the radius of the ball.

In a motor system, the outer raceway can be assumed stationary, since it is generally locked in place by an external casing, while the inner raceway is rotating at the speed of the shaft, i.e., $F_{o}=0$ and $F_{i}=F_{S}$. Therefore, in a motor system, (2)-(5) can be rewritten as

$$
\begin{aligned}
F_{C} & =\frac{1}{2} F_{S}\left(1-\frac{D_{b} \cos \theta}{D_{c}}\right) \\
F_{B P O} & =\frac{N_{B}}{2} F_{S}\left(1-\frac{D_{b} \cos \theta}{D_{c}}\right) \\
F_{B P I} & =\frac{N_{B}}{2} F_{S}\left(1+\frac{D_{b} \cos \theta}{D_{c}}\right) \\
F_{B} & =\frac{D_{c}}{2 D_{b}} F_{S}\left(1-\frac{D_{b}^{2} \cos ^{2} \theta}{D_{c}^{2}}\right) .
\end{aligned}
$$

Frequency-domain studies show that, when defects exist in a bearing, the defects will generate some of the above frequencies in the vibration signals. Many publications have discussed the use of these five frequencies to identify defects in a bearing assembly [5]-[7], [13], [23].

For defects on the raceway of a rolling bearing, each time a roller hits the defective raceway, the corresponding ball pass inner raceway frequency $F_{B P I}$ or ball pass outer raceway frequency $F_{B P O}$ will be excited. If the defective area is large, harmonics of $F_{B P I}$ or $F_{B P O}$ will also be present as an indication of the severity of the defects [5]-[7], [13], [23]. For defects existing on a bearing roller, usually, two times the ball rotational frequency $2 F_{B}$ will be generated. This is because the roller hits both the inner and outer raceways each time it spins on its own axis. In most cases, this frequency will be modulated with other existing frequencies, such as $F_{B P I}$ and $F_{B P O}$, resulting in a 
TABLE I

BEARING VIBRATION FEATURES

\begin{tabular}{|c|c|c|}
\hline Location of bearing defects & Frequency in Spectrum & Observations \\
\hline Good condition & $F_{S}, F_{B P O}, F_{B P I}$ & $\begin{array}{c}\text { Good condition can have } F_{S}, F_{B P O}, \\
F_{B P I} \text { and its harmonics, the amplitude } \\
\text { is small and even, no salient } \\
\text { frequency stands out. }\end{array}$ \\
\hline Bearing looseness & $F_{S}$ & $\begin{array}{l}\text { Looseness condition can have } F_{S}, \\
\text { and its harmonics. }\end{array}$ \\
\hline Rolling elements & $2 F_{B}, F_{B P O}, F_{B P I}$ & $\begin{array}{l}\text { For severe case these frequencies can } \\
\text { be modulated by } 2 F_{B}, F_{C} \text {, and the } \\
\text { natural frequency can be also excited. }\end{array}$ \\
\hline Bearing raceway & $F_{B P O}, F_{B P I}$ & $\begin{array}{l}\text { Increased severity of the defect results } \\
\text { in higher order harmonics being } \\
\text { produced, the frequency for the } \\
\text { raceway with defect will stand out, if } \\
\text { the clearance is small, } 2 F_{B} \text { can also } \\
\text { be presented. }\end{array}$ \\
\hline
\end{tabular}

more complicated spectrum. Occasionally, if the defective area on the roller is very large, the system natural frequency will also be excited and modulated with two times the ball rotational frequency [5]-[7], [13], [23]. Table I summarizes the possible motor bearing vibration features in the frequency domain.

\section{BEARING VibRATION FAUlt DiagnOSIS AlgORIthM USING NEURAL NETWORK}

In the design and study of motor bearing fault diagnosis schemes, it is important to determine if the designed fault diagnosis algorithm is able to correctly classify different bearing fault conditions. Using a well-controlled fault data environment, such as one obtained from a computer simulation, to verify the fault diagnosis algorithm's performance capability is essential. In this section, the authors use the Fast Prototype Motor Simulation software, MotorSim, [3], [24] to simulate and design the bearing vibration fault diagnosis algorithm.

MotorSim is a MATLAB-SIMULINK [25] based program that provides a framework for in-depth simulation of motor system dynamics. Although motor system dynamics simulation software cannot completely model all real-world situations, a computer simulation can assist in several aspects of motor system operation and control. MotorSim can be used to generate appropriate motor data, with different operating and loading conditions, in a cost-effective and time-efficient manner.

A significant amount of motor fault diagnosis research has focused on bearing vibration to detect motor bearing conditions. MotorSim can generate time-domain vibration signals for different conditions of bearing failure by incorporating a bearing wear submodule into the motor base module. Inverse
Fourier transform was used and fine tuned to reconstruct different bearing vibration time-domain signals based on those frequency features discussed in the previous section. Measurement noise was also introduced in the bearing vibration model. The results have been verified by comparison to real-world vibration signals [6]. Fig. 4 shows a bearing vibration signal generated by simulating bearing looseness and defects on the inner raceway and rolling element using MotorSim.

Table I shows that the vibration generated by defects in the bearing will show one, or some combination of several, of the five basic frequencies. Many publications [5]-[7], [13], [23] have discussed the usage of these five basic frequencies to identify defects in a bearing. Usually, an expert will examine the time-domain signal and the frequency spectra of bearing vibration to determine if there are any defects within the bearing. However, a problem with using experts for vibration analysis is that this experience, which is gained over a period of many years, is a very expensive and an inefficient use of resources. Therefore, if a neural network can be trained to emulate the knowledge of vibration experts, motor bearing fault diagnosis can be achieved more efficiently and at a reduced cost. The general structure of this neural-network-based motor bearing fault diagnosis system is shown in Fig. 5.

MotorSim is used to generate vibration signals with varying severity for each of three different bearing defects. The geometrical structure of the bearing is $D_{b}=0.5 \mathrm{in}, D_{c}=2.559$ in, and $N_{b}=9$. The three bearing defects are the vibrations caused by bearing looseness, by a circular defect located on the inner raceway with a variable size from the initial diameter of $0.03-0.225$ in at a severe case, and by a defect which resides on the rolling element increasing from 0.025 to $0.155 \mathrm{in}$. 


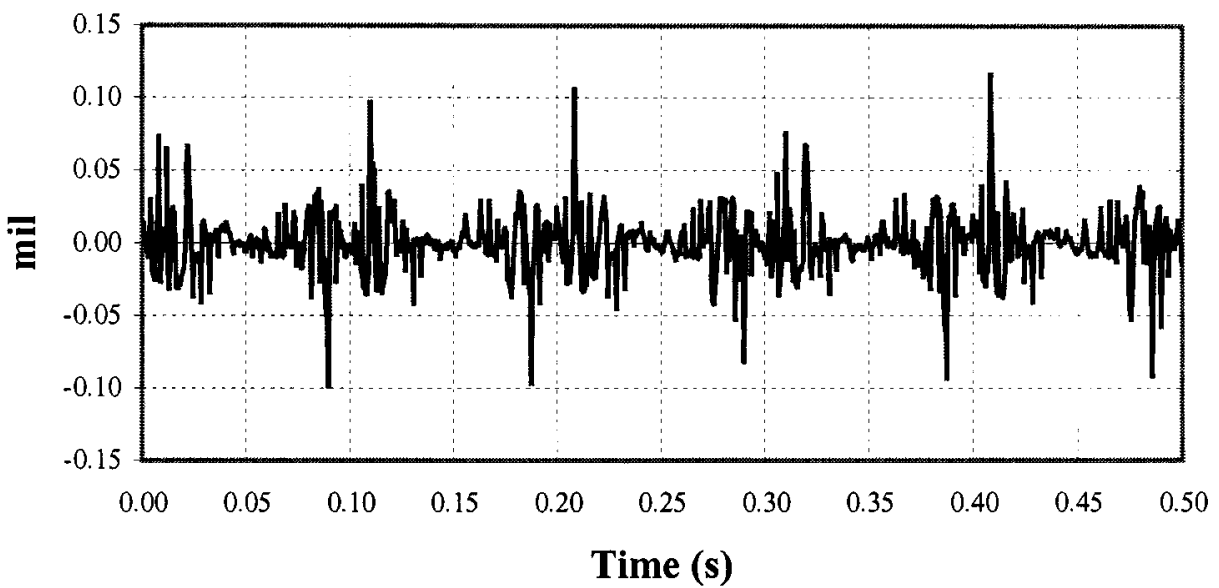

Fig. 4. Bearing vibration signal generated by MotorSIM.

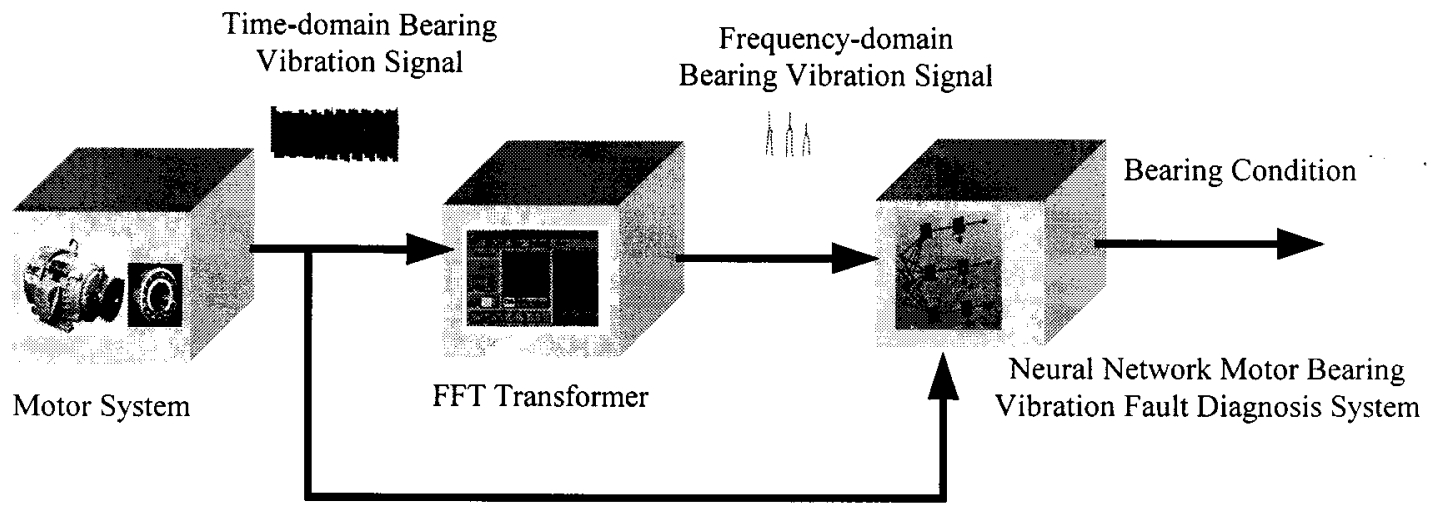

Fig. 5. Neural network motor bearing fault detection system.

Each signal consists of 2048 sampling points with a sampling time of 0.0005 seconds. An FFT is performed to extract the frequency characteristics of these vibration signals. This procedure is shown in

$$
D(k)=\sum_{n=0}^{N-1} d(n) e^{-j 2 \pi k n / N}, \quad k=0,1, \cdots, N-1
$$

where $N=2048$ and $d(n), 0 \leq n \leq N-1$ denotes the time-domain data generated by MotorSim. The power spectrum of the vibration signal is obtained as follows:

$$
\begin{aligned}
P(0) & =|D(0)|^{2}, \\
P(k) & =\left[|D(k)|^{2}+|D(N-k)|^{2}\right], \\
k=1,2, \cdots, \frac{N}{2}-1 & \\
P\left(\frac{N}{2}\right) & =\left|D\left(\frac{N}{2}\right)\right|^{2} .
\end{aligned}
$$

Next, we construct basic frequency signatures to represent different bearing vibrations. These signatures are created from the power spectrum of the vibration signal and consist of the corresponding basic frequencies, with varying amplitudes based on the defect present. Due to the energy leakage, when generating the signatures, we consider a frequency band of $5 \mathrm{~Hz}$ for each basic frequency. That is

$$
\text { frequency band }=[f-2.5, f+2.5]
$$

where $f$ is the basic frequency.

For the different characteristic frequencies, the frequency amplitude can be represented as

$$
A(F)=\sum_{F_{k}=f-2.5}^{f+2.5} P\left(\left\lceil\frac{F_{k}}{\Delta f}\right\rceil\right), \quad \Delta f=\frac{1}{N T_{s}}
$$

where $T_{s}$ is the sampling time, and $N$ is the total sampling points used.

Therefore, the amplitude of spectrum for frequencies $F_{S}$, $F_{B P O}$, and $F_{B P I}$ are

$$
\begin{aligned}
x_{F_{S}} & =A\left(F_{S}\right) \\
x_{F_{B P O}} & =A\left(F_{B P O}\right) \\
x_{F_{B P I}} & =A\left(f_{B P I}\right) .
\end{aligned}
$$

The time-domain information considered is the maximum and mean value of the amplitude of vibration waveform, and 
the Kurtosis factor of the vibration waveform. They are defined as

$$
\begin{aligned}
A_{\max } & =\max _{n=0}^{N-1}(d(n)) \\
A_{\text {mean }} & =\frac{1}{N} \sum_{n=0}^{N-1} d(n) \\
K_{f} & =\frac{\frac{1}{N} \sum_{n=0}^{N-1}(d(n))^{4}}{D_{\mathrm{rms}}}
\end{aligned}
$$

where

$$
D_{\mathrm{rms}}=\sqrt{(1 / N) \sum_{n=0}^{N-1} d^{2}(n)}
$$

The completed neural network will have six input measurements

$$
X=\left[A_{\max }, A_{\text {mean }}, K_{f}, x_{F_{S}}, x_{F_{B P O}}, x_{F_{B P I}}\right] .
$$

The neural network has three outputs, each one serving as an indicator for one of the three fault conditions (bearing looseness, defects on the inner raceway, and defects on the rolling element) producing an output of 1 for good and -1 for bad.

Different neural network configurations have been evaluated to determine the possible structure of the motor rolling bearing fault diagnosis algorithm. The performance of the final motor bearing fault diagnosis machine, a three-layer feedforward neural network with ten hidden nodes, was tested using the data generated by MotorSim and is summarized in Table II. A learning rate of 0.01 and momentum of 0.8 was selected for all cases based on the experience of the authors. Table II shows that the neural network was able to accurately detect the three faults.

\section{REAL-TIME DATA VERIFICATION}

In this section, actual motor rolling bearing vibration data were collected to verify the feasibility of applying a neural network to diagnose bearing fault. The vibration data was generated with a Machinery Fault Simulator manufactured by SpectraQuest, Richmond, VA, 1997. The Machinery Fault Simulator allows for the measurement of vibration data with the motor operating under a variety of fault conditions. The platform of the Machinery Fault Simulator is depicted in Fig. 6.

The Machinery Fault Simulator can simulate different types of motor faults. Bearing fault vibration is generated by replacing the front bearing with a bearing of known fault condition provided by the bearing manufacturer. The vibration signals are measured through two vibration sensors located on the $X$ and $Y$ axes. The motor speed or the shaft rotational frequency $F_{S}$ can be collected from the encoder attached on the motor shaft. The signals from the vibration sensors and encoder are transmitted to a National Instruments SCXI chassis [26] for signal conditioning and then sampled at a rate of 20000 samples/s via a National Instruments A/D PC card. Each data set collected contains 16384 samples. An FFT of the data set is followed immediately
TABLE II

Performance of the Neural Network Motor Bearing Fault Diagnosis Algorithm ON Simulated Motor Rolling BEARING FAUlt DATA

\begin{tabular}{c|c|c|c}
\hline \multicolumn{2}{c|}{$\begin{array}{c}\text { Bearing } \\
\text { Fault }\end{array}$} & $\begin{array}{c}\text { Number of } \\
\text { Data Sets }\end{array}$ & Diagnosis Results \\
\hline \multirow{3}{*}{ Looseness } & Good & 72 & Correct detect \\
\cline { 2 - 4 } & Bad & 72 & 71 \\
\cline { 2 - 4 } & Total & $\mathbf{1 4 4}$ & $\mathbf{1 3 9 / 9 6 . 5 \%}$ \\
\hline \multirow{3}{*}{ Inner Race } & Good & 72 & 58 \\
\hline \multirow{3}{*}{ Ball } & Total & $\mathbf{1 4 4}$ & $\mathbf{1 2 7 / 8 8 . 2 \%}$ \\
\hline & Good & 72 & 71 \\
\cline { 3 - 4 } & Bad & 72 & 70 \\
\cline { 3 - 4 } & Total & $\mathbf{1 4 4}$ & $\mathbf{1 4 1 / 9 7 . 9 \%}$ \\
\hline
\end{tabular}

after finishing the data collection and the result is saved on the hard disk. The overall data acquisition system setup is shown in Fig. 7.

Three kinds of bearing fault vibrations are generated using the Machinery Fault Simulator. They are: 1) defect on bearing ball; 2) defect on inner raceway; and 3) defect on outer raceway. In addition, the normal bearing vibration is also measured in order to compare with the fault cases. The geometrical structure of the bearing used in the experiment is $D_{b}=0.3125 \mathrm{in}, D_{c}=$ $1.319 \mathrm{in}$, and $N_{b}=8$. The faulted bearings are provided by the manufacturer; the detailed information (size and geometry) for the defects are not known.

The vibration signals under normal condition, bearing ball defect, and inner raceway and outer raceway defects are measured at five shaft rotational frequencies $F_{S}$, which are 15, 57.5, 25, 40 , and $32 \mathrm{~Hz}$, respectively. At each shaft frequency, five measurements are made for each working condition.

As in the real-world environment, the motor speed cannot keep rotating at a constant $F_{S}$ precisely. This fluctuation is caused from external factors such as the performance of the controller, noise, and disturbance in power system. The fluctuation in $F_{S}$ also causes other bearing basic frequencies to deviate from the calculated value. Therefore, the frequency band range in (14) needs to be adjusted. Thus, in each measurement, the deviation of $F_{S}$ is computed by

$$
D_{\mathrm{dev}}=\max \left(F_{s}\right)-\min \left(F_{s}\right) .
$$

Generally, the deviation in each measurement under the same bearing fault condition may not be identical. In order to derive the range of the deviations for all conditions, the maximum value of deviation from all measurements in each fault condition is considered. The maximum deviation at each $F_{S}$ under different conditions is plotted in Fig. 8 .

The frequency band range has to be wide enough to cover the maximum deviation in every condition. Therefore, the variable range is defined by drawing the $V$-range line shown in Fig. 8 from the maximum deviation of every fault condition at $F_{S}=$ $15 \mathrm{~Hz}$ to the maximum deviation at $F_{S}=32 \mathrm{~Hz}$ because this 


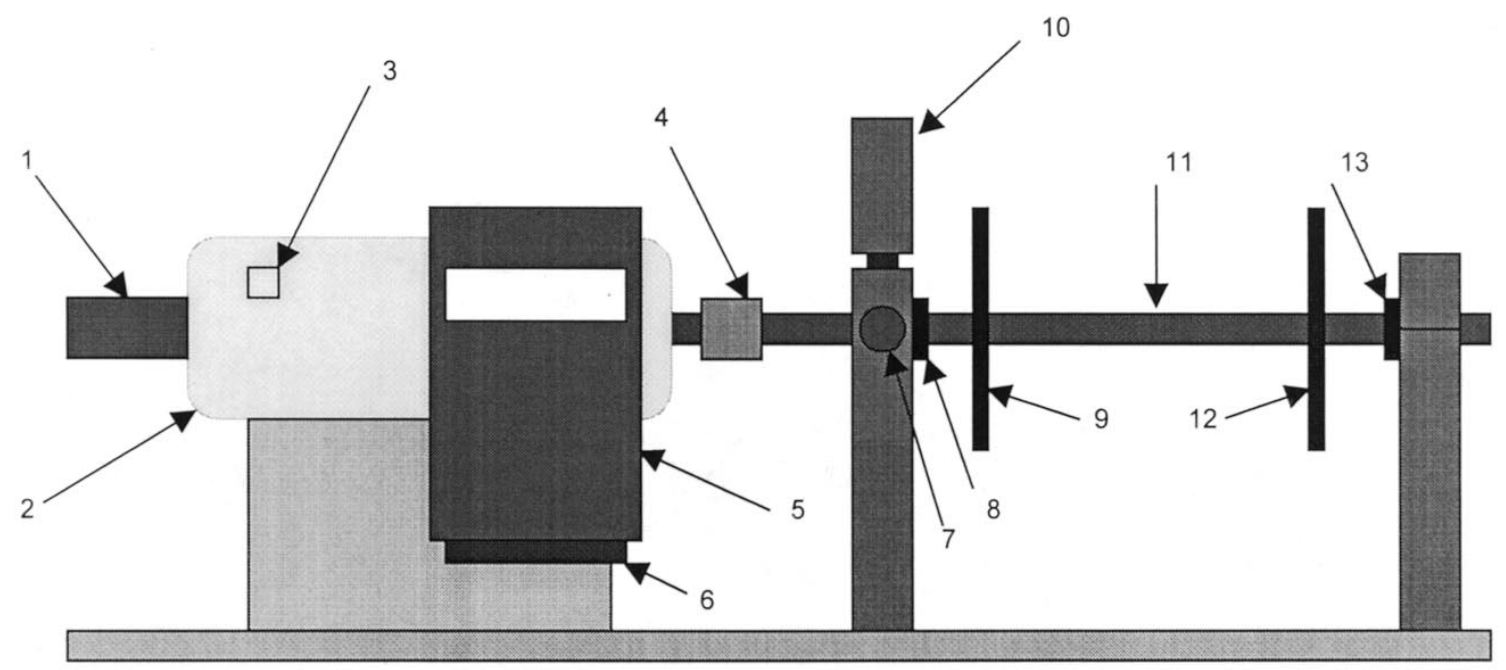

Fig. 6. Motor fault simulator drawing (1: encoder; 2: three-phase induction motor; 3: temperature sensor; 4: shaft coupling; 5: variable-frequency motor drive; 6: current sensor; 7: $x$-axis vibration sensor; 8: front bearing; 9: rotational disk 1; 10: $y$-axis vibration sensor; 11: motor shaft; 12: rotational disk 2; 13: rear bearing).

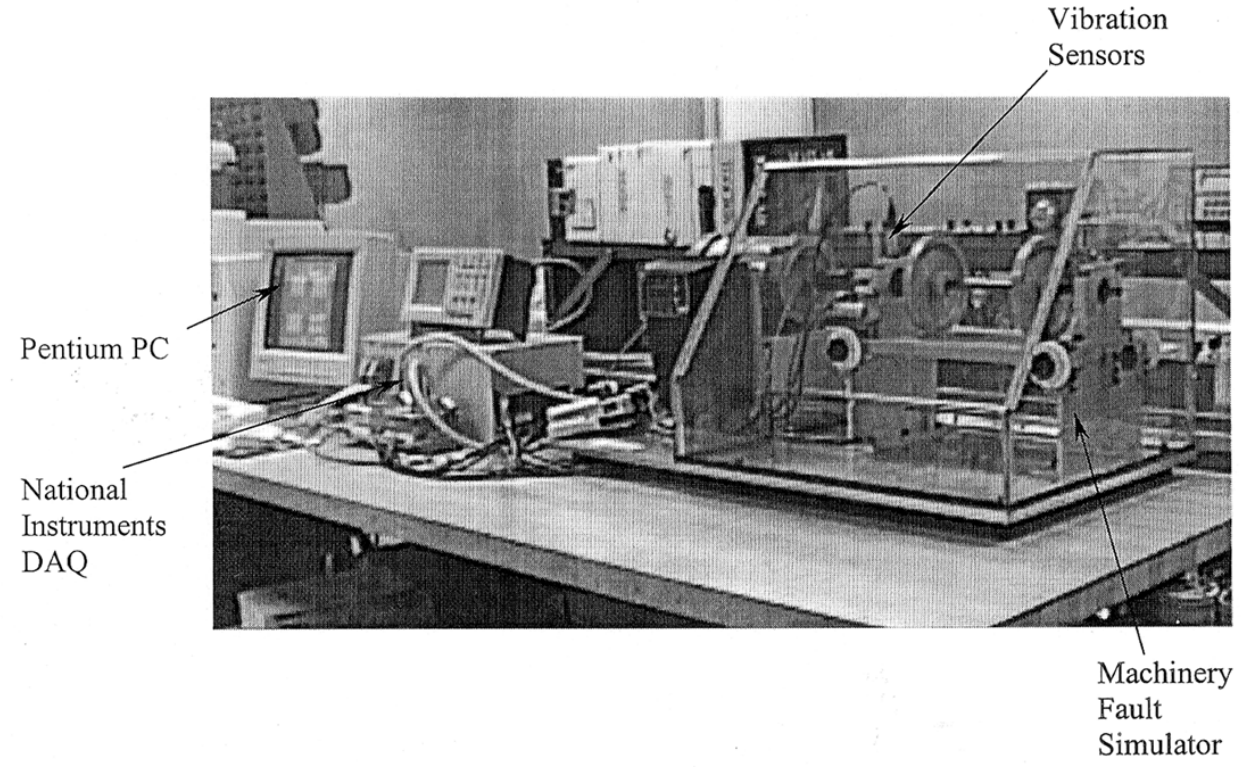

Fig. 7. Real-time motor bearing vibration data acquisition system.

range represents the maximum proportion between $F_{S}$ and deviation. This line is extended to cover other $F_{S}$. The slope of this line is used to define the frequency band range. Thus, (15) becomes

$$
\begin{aligned}
A(F) & =\sum_{F_{k}=f-R}^{f+R} P\left(\left\lceil\frac{F_{k}}{\Delta f}\right\rceil\right) \\
R & =\frac{1}{2}\left(\alpha(f-15)+\max _{F_{s}=15}\left(D_{\mathrm{dev}}\right), f \geq 15\right)
\end{aligned}
$$

where $\alpha$ is the slope of the $V$-range line.

The modified frequency band range from (24) provides a more robust scheme to process the measured data described by the equations in Section III. As the real-time environment cannot provide a vibration signal under different severity for one bearing element defect as we did in the simulation data, vibration signals generated from faulty bearings at different motor speeds are examined instead. To examine the effect of motor speed on the performance of diagnosis of the neural network motor bearing fault scheme, two neural networks are designed in this verification. The input for these neural networks are $X=\left[A_{\max }, A_{\text {mean }}, K_{f}, x_{F_{S}}, x_{F_{B P O}}, x_{F_{B P I}}\right]$ and $X=\left[F_{S}, A_{\max }, A_{\text {mean }}, K_{f}, x_{F_{S}}, x_{F_{B P O}}, x_{F_{B P I}}\right]$ correspondingly. The $F_{S}$ used to compute $x_{F_{S}}, x_{F_{B P O}}, x_{F_{B P I}}$ is the average $F_{s}$ from each measurement. The training of the neural network with real-time data sets is performed by the MATLAB Neural Networks Toolbox 3.0 [27]. A three-layer feedforward neural network is trained by using the Levenberg-Marquardt algorithm. The activation functions at the hidden layer and output layer in the network are a hyperbolic tangent function. Two parameters, the number of hidden 

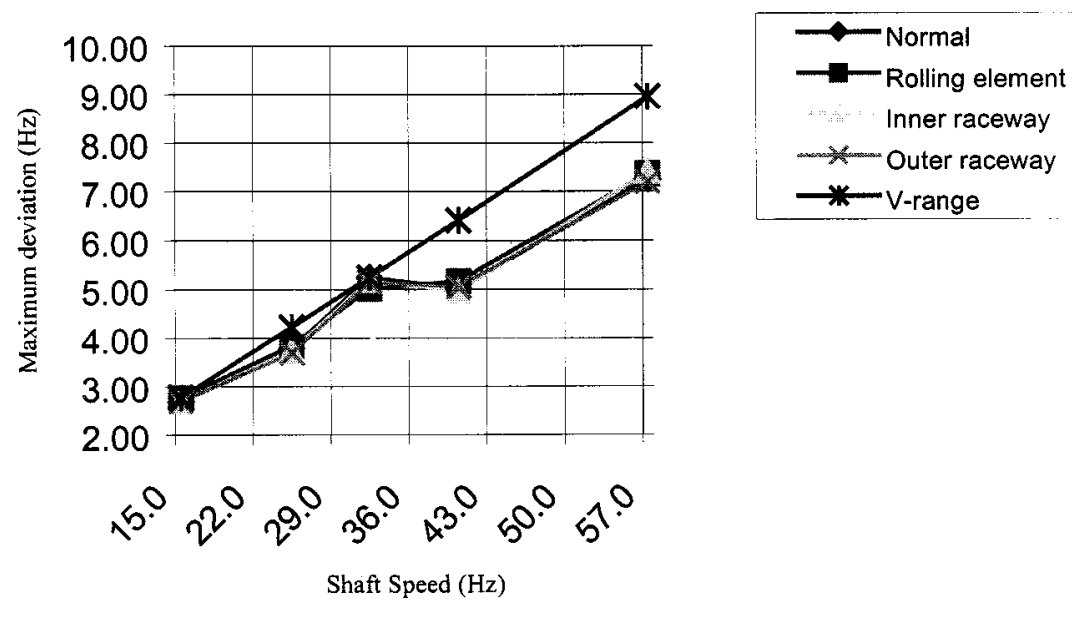

Fig. 8. Frequency fluctuation in real-time measurement.

TABLE III

Performance of Real-Time neural Network Motor Bearing Fault Diagnosis Algorithm

\begin{tabular}{|c|c|c|c|c|c|c|c|c|c|c|c|}
\hline \multirow{3}{*}{$\begin{array}{l}\text { Hidden } \\
\text { Neuron }\end{array}$} & \multirow{3}{*}{ Learning rate } & \multirow{3}{*}{$\begin{array}{l}\text { With } \\
\text { speed input }\end{array}$} & \multicolumn{9}{|c|}{ Correct detection } \\
\hline & & & \multicolumn{2}{|c|}{ Normal } & \multicolumn{2}{|c|}{ Rolling Elements } & \multicolumn{2}{|c|}{ Inner raceway } & \multicolumn{2}{|c|}{ Outer raceway } & \multirow{2}{*}{$\begin{array}{c}\text { Average } \\
\text { Detection rate }\end{array}$} \\
\hline & & & Number & Accuracy & Number & Accuracy & Number & Accuracy & Number & Accuracy & \\
\hline 10 & 0.001 & Yes & 40 & 100 & 38 & 95 & 33 & 82.5 & 36 & 90 & 91.875 \\
\hline 15 & 0.001 & Yes & 39 & 97.5 & 39 & 97.5 & 36 & 90 & 37 & 92.5 & 94.375 \\
\hline 20 & 0.001 & Yes & 39 & 97.5 & 39 & 97.5 & 37 & 92.5 & 36 & 90 & 94.375 \\
\hline 25 & 0.001 & Yes & 40 & 100 & 39 & 97.5 & 34 & 85 & 39 & 97.5 & 95 \\
\hline 30 & 0.001 & Yes & 40 & 100 & 37 & 92.5 & 33 & 82.5 & 38 & 95 & 92.5 \\
\hline 35 & 0.001 & Yes & 40 & 100 & 40 & 100 & 33 & 82.5 & 38 & 95 & 94.375 \\
\hline 10 & 0.005 & Yes & 39 & 97.5 & 38 & 95 & 36 & 90 & 37 & 92.5 & 93.75 \\
\hline 15 & 0.005 & Yes & 40 & 100 & 40 & 100 & 36 & 90 & 37 & 92.5 & 95.625 \\
\hline 20 & 0.005 & Yes & 40 & 100 & 40 & 100 & 34 & 85 & 36 & 90 & 93.75 \\
\hline 25 & 0.005 & Yes & 40 & 100 & 40 & 100 & 35 & 87.5 & 39 & 97.5 & 96.25 \\
\hline 30 & 0.005 & Yes & 40 & 100 & 39 & 97.5 & 35 & 87.5 & 37 & 92.5 & 94.375 \\
\hline 35 & 0.005 & Yes & 39 & 97.5 & 39 & 97.5 & 34 & 85 & 37 & 92.5 & 93.125 \\
\hline 10 & 0.001 & No & 40 & 100 & 39 & 97.5 & 33 & 82.5 & 32 & 80 & 90 \\
\hline 15 & 0.001 & No & 39 & 97.5 & 35 & $87 . \overline{5}$ & 37 & 92.5 & 31 & 77.5 & 88.75 \\
\hline 20 & 0.001 & No & 40 & 100 & 38 & 95 & 35 & 87.5 & 35 & 87.5 & 92.5 \\
\hline 25 & 0.001 & No & 40 & 100 & 40 & 100 & 33 & 82.5 & 35 & 87.5 & 92.5 \\
\hline 30 & 0.001 & No & 39 & 97.5 & 39 & 97.5 & 32 & 80 & 30 & 97.5 & 93.125 \\
\hline 35 & 0.001 & No & 38 & 95 & 33 & 82.5 & 36 & 90 & 31 & 77.5 & 86.25 \\
\hline 10 & 0.005 & No & 39 & 97.5 & 38 & 95 & 30 & 75 & 32 & 80 & 86.875 \\
\hline 15 & 0.005 & No & 38 & 95 & 38 & 95 & 30 & 75 & 36 & 90 & 88.75 \\
\hline 20 & 0.005 & No & 39 & 97.5 & 39 & 97.5 & 32 & 80 & 36 & 90 & 91.25 \\
\hline 25 & 0.005 & No & 39 & 97.5 & 32 & 80 & 33 & 82.5 & 35 & 87.5 & 86.875 \\
\hline 30 & 0.005 & No & 40 & 100 & 40 & 100 & 35 & 87.5 & 30 & 75 & 90.625 \\
\hline 35 & 0.005 & No & 40 & 100 & 40 & 100 & 33 & 82.5 & 34 & 85 & 91.875 \\
\hline
\end{tabular}

neurons and the learning rate, are varied to find the optimal design. The different numbers of hidden neurons applied in the verification are $10,15,20,25,30$, and 35 . The two learning rates used are 0.001 and 0.005 . All inputs are normalized to be in the range $[-1,1]$ before being applied to train the network. To improve generalization and the avoid overtraining problem, the cross-validation method [28] is applied during the training.

Table III shows the performance of the real-time neural network motor bearing fault diagnosis scheme. There are totally 40 real-time testing data sets to test the accuracy of the trained neural network to diagnose different motor bearing faults. The result demonstrates that with proper processing of the measured data and possible training procedure, the neural network motor bearing fault diagnosis schema can diagnose bearing faults with desired accuracy.

\section{CONCLUSION}

This paper has discussed several popular rolling bearing vibration features in both the time and frequency domain and the use of signal processing to provide features to be used for bearing fault diagnosis. Neural networks have been used in this paper to perform motor bearing fault diagnosis based on the extracted information features. Computer-simulated data were first used to study and design the neural network motor bearing fault diagnosis algorithm. Actual bearing vibration data collected in real-time were then applied to perform initial testing and validation of the approach. The results show that neural networks can be effectively used in the diagnosis of various motor bearing faults through appropriate measurement and interpretation of motor bearing vibration signals. 


\section{ACKNOWLEDGMENT}

The authors would like to thank SpectraQuest Inc. for its donation of the Machinery Fault Simulator.

\section{REFERENCES}

[1] M.-Y. Chow, Y.-S. Lee, and H. J. Trussell, "Motor incipient fault detection using artificial neural network and fuzzy logic technologies," in Computer Aided Maintenance, Methodology and Practices, J. Lee, Ed. London, U.K.: Chapman \& Hall, 1996.

[2] M.-Y. Chow and S.-O. Yee, "An adaptive backpropagation through time training algorithm for a neural controller," in Proc. IEEE Int. Symp. Intelligent Control, 1991, pp. 170-175.

[3] M.-Y. Chow, Methodologies of Using Neural Network and Fuzzy Logic for Motor Incipient Fault Detection. Singapore: World Scientific, 1997.

[4] K. Ragulskis and A. Yurkauskas, Vibration of Bearings. Bristol, PA: Hemisphere, 1989.

[5] G. Lipovszky, K. Solyomvari, and G. Varga, Vibration Testing of Machines and Their Maintenance. Amsterdam, The Netherlands: Elsevier, 1990.

[6] J. I. Taylor, The Vibration Analysis Handbook. Tampa, FL: Vibration Consultants, 1994.

[7] T. A. Harris, Rolling Bearing Analysis. New York: Wiley, 1991.

[8] H. Ohta and N. Sugimoto, "Vibration characteristics of tapered roller bearings," J. Sound Vib., vol. 190, no. 2, pp. 137-147, 1996.

[9] A. Palmgren, Ball and Roller Bearing Engineering. Philadelphia, PA: Burbank, 1959.

[10] P. Eschmann, L. Hasbargen, and K. Weigand, Ball and Toller Bearings-Their Theory, Design, and Application. London, U.K.: Heyden, 1958 .

[11] J. C. Robinson, R. G. Canada, and K. R. Piety, "PeakVue analysis-New methodology for bearing fault detection," Sound Vib., vol. 30, no. 11, pp. 22-25, Nov. 1996.

[12] C. Jackson, The Practical Vibration Primer. Houston, TX: Gulf, 1979.

[13] S. Korablev, V. Shapin, and Y. Filatov, Vibration Diagnostics in Precision Instruments. Bristol, PA: Hemisphere, 1989.

[14] M. Donley, W. Stokes, G. S. Jeong, K. K. Suh, and S. G. Jung, "Validation of finite element models for noise/vibration/harshness simulation," Sound Vib., vol. 30, no. 8, pp. 18-23, Aug. 1996.

[15] R. A. Collacott, Vibration Monitoring and Diagnosis. New York: Halsted, 1979.

[16] A. Dimarogonas, Vibration for Engineers. Englewood Cliffs, NJ: Prentice-Hall, 1996.

[17] M.-Y. Chow, R. N. Sharpe, and J. C. Hung, "On the application and design consideration of artificial neural network fault detectors," IEEE Trans. Ind. Electron., vol. 40, pp. 181-198, Apr. 1993.

[18] M.-Y. Chow, A. V. Chew, and S. O. Yee, "Performance of a fault detector artificial neural network using different paradigms," in Proc. SPIE Applications of Artificial Neural Networks III Conf., 1992.

[19] B. Li, G. Goddu, and M.-Y. Chow, "Detection of common motor bearing faults using frequency-domain vibration signals and a neural network based approach," in Proc. American Control Conf., 1998, pp. 2032-2036

[20] S. Lu, "A transfer matrix method for nonlinear vibration analysis of rotor-bearing systems," presented at the 1991 ASME Design Tech. Conf.-13th Biennial Conf. Mechanical Vibration and Noise, Miami, FL, Sept. 22-25, 1991, pp. 75-83.

[21] N. F. Rieger and J. F. Crofoot, Vibrations of Rotating Machinery. Rochester, NY: Rochester Inst. of Technol., 1977.

[22] J. Strader, "A vibration mathematics primer," Sound Vib., vol. 29, no. 6, pp. 18-23, June 1995.

[23] J. Raymond and A. Guyer, Rolling Bearings Handbook and Troubleshooting Guide. Radnor, PA: Chilton, 1996.

[24] M.-Y. Chow, S. Altug, and B. Li, "Motor system time domain fast prototype software simulation," in Proc. Nat. Symp. Electric Machines, 1997, pp. 183-187.

[25] SIMULINK-Dynamic System Simulation for MATLAB, The MathWorks Inc., Natick, MA, 1998.

[26] Instrumentation Reference and Catalogue, National Instruments, Austin, TX, 1997.

[27] H. Demuth and M. Beale, User's Guide for Nuural Network Toolbox for Use with MATLAB. Natick, MA: The MathWorks Inc., 1998.
[28] S. Haykin, Neural Networks-A Comprehensive Foundation. Englewood Cliffs, NJ: Prentice-Hall, 1999.

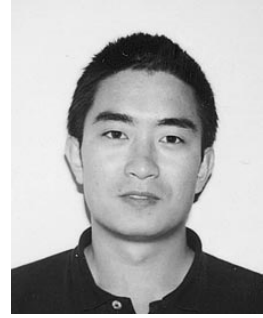

Bo Li (S'99) received the B.S. and M.S. degrees in electrical engineering from Tsinghua University, Beijing, China, in 1990 and 1993, respectively. He is currently working toward the Ph.D. degree in the Department of Electrical and Computer Engineering, North Carolina State University, Raleigh.

He was an Electrical Engineer with the Electrical Power Planning and Engineering Institute of China for several years.

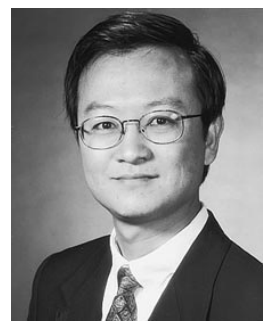

Mo-Yuen Chow (S'81-M-82-SM'93) received the B.S. degree from the University of Wisconsin, Madison, and the M.Eng. and Ph.D. degrees from Cornell University, Ithaca, NY, in 1982, 1983, and 1987, respectively, all in electrical engineering.

Upon completion of the Ph.D. degree, he joined the faculty of North Carolina State University, Raleigh, where he is currently a Professor in the Department of Electrical and Computer Engineering. He has also been a Consultant to Duke Power Company, Otis Elevator Company, Taiwan Power Company, J. W. Harley Company, and was a Faculty Intern at Duke Power Company. $\mathrm{He}$ is working in the areas of control and networking. His core technologies are diagnosis and control, artificial neural networks, and fuzzy logic. Since 1987, he has been involved in the areas of motors, process control, power systems, and communication systems. He has served as a Principal Investigator in several projects supported by the National Science Foundation, Center for Advanced Computing and Communication, Nortel Company, Electric Power Research Institute, Duke Power Company, ABB Company, Electric Power Research Center, and Army Construction Engineering Research Laboratory. He established the Advanced Diagnosis and Control Laboratory at North Carolina State University. He has authored one book, several book chapters, and more than 80 journal and conference articles related to his research work. He is the past President of the Triangle Area Neural Network Society.

Dr. Chow served as a Guest Editor for the IEEE TRANSACTIONS ON InDUSTRIAL ELECTRONICS Special Issue on Application of Intelligent Systems to Industrial Electronics. $\mathrm{He}$ is currently the IEEE Industrial Electronics Society Vice President for Member Activities, an Associate Editor of the IEEE TRANSACTIONS ON INDUSTRIAL ElECTRONICS, an AdCom Member of the IEEE Industrial Electronics Society, an AdCom Member of the IEEE Neural Networks Council, Chairman of the IEEE Neural Networks Council Regional Interest Group Committee, and past Editor-in-Chief of the IEEE Industrial Electronics Society Newsletter. In addition, he is listed in Who's Who Among Asian Americans.

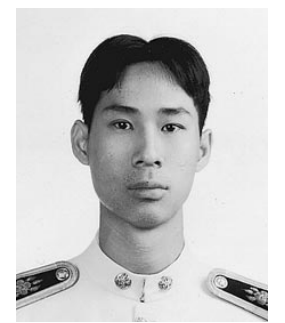

Yodyium Tipsuwan (S'99) was born in Chiangmai, Thailand. He received the B.Eng. degree (with honors) in computer engineering in 1996 from Kasetsart University, Bangkok, Thailand, and the M.S. degree in electrical engineering in 1999 from North Carolina State University, Raleigh, where he is currently working toward the Ph.D. degree in distributed-network-based control systems.

From 1996 to 1997, he was a Lecturer at Kasetsart University, where he taught digital system design and microprocessor-based control systems. During his M.S. studies, he focused on intelligent control and its implementation. His current research interests include mechatronics and computational intelligence and its distributed-network-based control. 


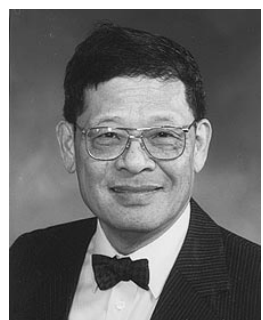

James C. Hung (S'55-M'60-SM'62-F'84) received the B.S. degree in electrical engineering from $\mathrm{Na}-$ tional Taiwan University, Taipei, Taiwan, R.O.C., and the M.E.E. and Eng.Sc.D. degrees from New York University, New York, NY.

From 1956 to 1961, he was an Instructor in the Department of Electrical Engineering, New York University. In 1961, he joined the University of Tennessee, Knoxville, as an Assistant Professor. $\mathrm{He}$ became an Associate Professor and a Professor in 1962 and 1965, respectively. He is a specialist in system analysis, system design, and data processing, with applications to navigation, guidance, and control. He has been a Consultant to the U.S Government and to industry.

Dr. Hung is a member of Sigma Xi, Tau Beta Pi, Eta Kappa Nu, and Phi Kappa Phi. He is a Registered Professional Engineer in the State of Tennessee. He is active in the IEEE Industrial Electronics Society (IES), having been the General Chair of three international conferences, Track Chair of several IECONs, the Editor-in-Chief of the IEEE TRANSACTIONS ON INDUSTRIAL ELECTRONICS for five years, and the IES Vice-President for small conferences in 1997. He has also served as President of the IES and as a Member of the IEEE Technical Activity Board for 1998-1999. He was awarded the IEEE Anthony J. Hornbeck Service Award in 1995 and the IEEE Third Millennium Medal in 2000. He was named Distinguished Service Professor of the University of Tennessee, Knoxville, in 1984. He has received several awards and citations from the University of Tennessee and the U.S. Government. He has been named an Honorary Professor by Nanjing University of Aeronautics and Astronautics, South China University of Technology, and Hunan University. 